\title{
COMBINED ASK/FSK AND ASK/DPSK MODULATION FORMATS FOR OPTICALLY LABELED SIGNALS
}

\author{
Sulur ${ }^{1}$, Ton Koonen ${ }^{1}$, Idelfonso Tafur Monroy ${ }^{1}$, Jean Jennen ${ }^{2}$, Huug de \\ Waardt ${ }^{1}$, Geert Morthier ${ }^{3}$ \\ ${ }^{1}$ COBRA Institute, Eindhoven University of Technology, The Netherlands \\ ${ }^{2}$ Bell laboratories, Lucent Technologies NL, Huizen, The Netherlands \\ ${ }^{3}$ IMEC, University of Ghent, Belgium
}

\begin{abstract}
A novel two-level optical labeling method for highly efficient transport of IP packets through WDM networks is proposed. By assigning both a wavelength label and a labeling DPSK or FSK format (orthogonal to the ASK format of the data payload), packets can be routed transparently through end-to-end optical paths. The design of a dual-label controlled optical router is presented, which features label swapping and multicasting. Key element for the label swapping is an optically integrated wavelength converter. The performance of combined ASK/DPSK and ASK/FSK modulation formats is analyzed and compared. In comparison with the combined ASK/DPSK modulation formats, the combined ASK/FSK modulation format is easier to implement and it tolerates a much larger laser line-width, but also requires a more careful fiber dispersion compensation in order to reduce the impact of the phase-to-intensity conversion. This work is part of the new IST project STOLAS - Switching Technologies for Optically Labeled Signals.
\end{abstract}

Key words: Optical communications, WDM network, MPLS, GMPLS, optical networks, optical label switching.

\section{INTRODUCTION}

Telecommunication networks are experiencing an explosive growth of packet-based data traffic, fueled by Internet usage. The past twelve months have shown a tripling of the worldwide Internet traffic. Putting IP packets 
directly into WDM channels, thus skipping the SDH and ATM transport layers, can yield a significant improvement of the network throughput. The recently suggested MP $\lambda$ S protocol enables IP-over-WDM, by providing wavelength-switched channels in a similar way as label-switched paths in the MPLS protocol [1]. In the near future, optical networks will operate at Tbit/s line rates, and optical routing of IP packets of which the majority has quite a small size ( $50 \%$ is less than 522 bytes) requires packet forwarding with very low latency. With Optical Label Switching, packets are marked with an optical label (e.g. a wavelength), which may be swapped in each network node [2]. Thus data packets can be routed transparently in the optical domain via end-to-end optical paths, aided by their optical label; they can bypass the electronic switching in the network core routers, thus significantly increasing the network's throughput. With the pervasive usage of IP-based devices, multiple optical addressing levels would be welcome for advanced routing and traffic engineering. Therefore, in addition to labeling with a wavelength, a second level of optical labeling is proposed in the IST STOLAS project [3], by modulating label information in a DPSK (or FSK) format which is orthogonal to the $A S K$ format used for the payload data. The rest of this paper is organised as follows. First, the design of a dual-label controlled optical router is presented. Second, the architecture of the two-label swapper node is presented. Third, the performance of the combined ASK/FSK and ASK/DPSK modulation format for label coding is analysed and discussed. A comparison of the performance of both modulation schemes is given. Finally, conclusion is drawn.

\section{LABEL-CONTROLLED OPTICAL PACKET ROUTING}

In an optically routed packet transport network, packets entering the network from an underlying access or metropolitan network first need to get an optical label at the entrance point, the edge router. The two-level optical labeling proposed in this paper is done in two steps; see Fig. 1. The incoming IP packets are buffered and aggregated, and a label setting circuit determines the wavelength label to be assigned. The continuous wave output light of a fast tunable laser diode is amplitude modulated with low chirp in an external modulator by the IP packet payload data. Subsequently, the nextlevel label information is impressed in the orthogonal DPSK format by means of a phase modulator. A payload data rate of $10 \mathrm{Gbit} / \mathrm{s}$ is envisaged, and a label data rate of $155 \mathrm{Mbit} / \mathrm{s}$. Scrambling of the payload data (e.g., with a $16^{\text {th }}$ order polynom, following ITU-T Rec. G.709) should provide enough bit transitions to be DPSK modulated. An alternative approach is to 
deploy FSK modulation. Again, the tunable laser needs to be set to the appropriate wavelength label, but the additional label information now can be attached by direct current modulation of the laser, delivering an FSKmodulated signal to the amplitude modulator. Thus a separate phase modulator is avoided; however, the line signal exhibits more chirp which will affect the transmission performance more.

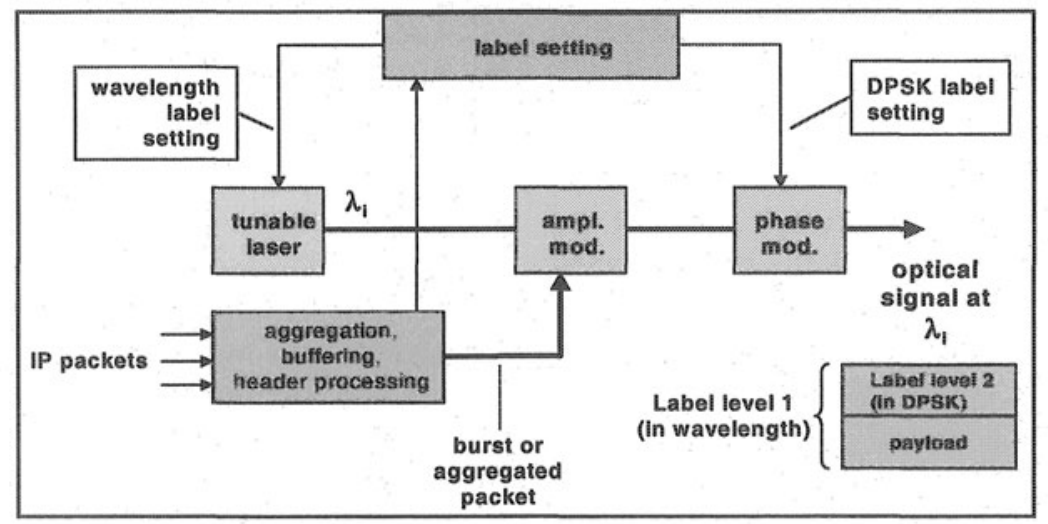

Figure 1. Two-level optical labeling in edge router.

In the network routing nodes, both the wavelength and the DPSK (or FSK) label are inspected, and new labels are set with the aid of a routing table. The general setup of such a label-controlled router is shown in Fig. 2. It enables routing of packets in any of the $N$ wavelength channels at any of the $M$ fibre input lines to any wavelength channel at any fibre output line. At every inlet a wavelength demultiplexer separates the individual wavelength channels, and subsequently in a wavelength-and-phase information swapper a new two-level label is assigned to each packet as commanded by the routing table. In an Arrayed Waveguide Grating Router (AWGR), by means of its wavelength the packet is directed to the appropriate output port. To avoid packet collision when two or more packets are contending for the same output port, a packet can be buffered in a variable delay line. As illustrated in Fig. 3, 8 distinct delay times can be stepwise set with a threestage switchable delay line using fibre delay loops with length ratios 4:2:1.

The router setup also provides means for multicasting packets optically. By assigning the appropriate wavelength, a packet emerges at a multicast outlet of the AWGR, from where it is fed via a power splitter to a number of label swappers at the inputs of the AWGR, by which the packet is multicasted to the appropriate output fibers. 


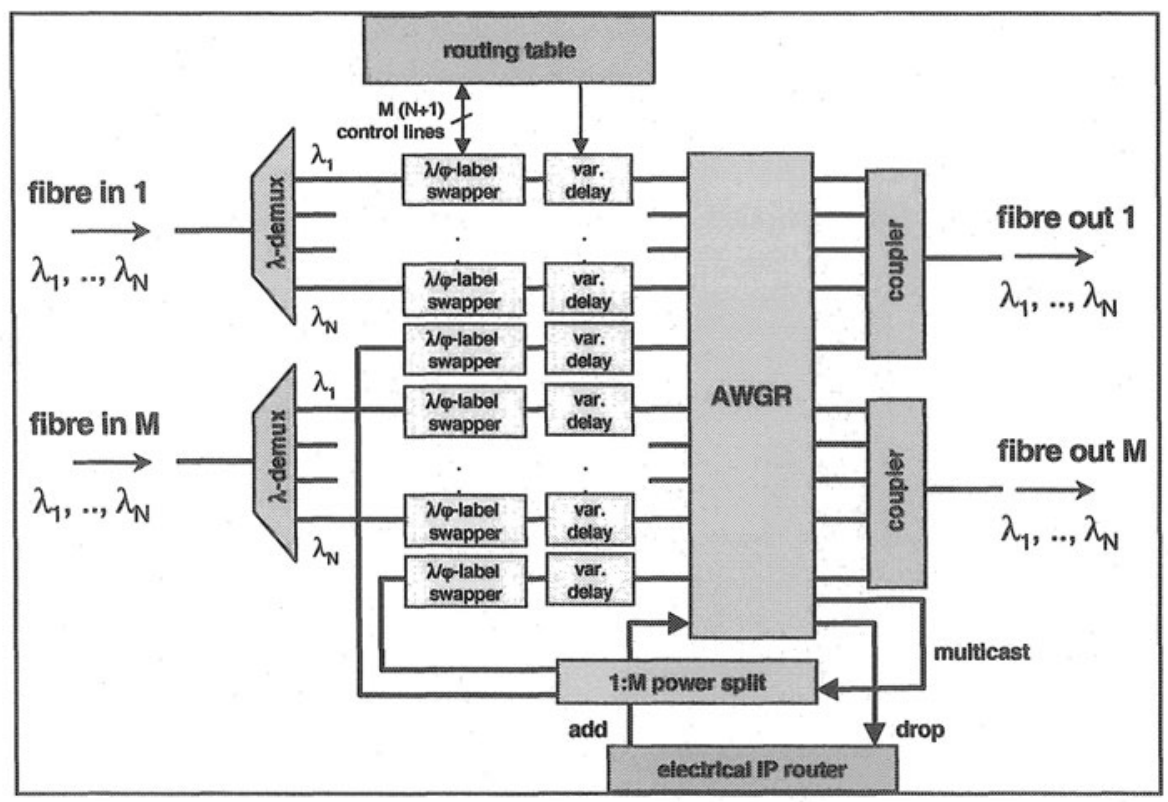

Figure 2. Optical label-controlled router.

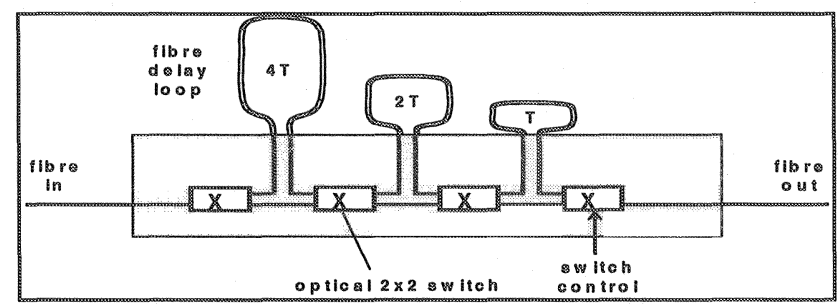

Figure 3. Switchable delay line

For a router handling 4 wavelengths and having 2 input and 2 output fibres, a $12 \times 12$ AWGR would be needed. The same functionality, however, can be obtained with three $4 \times 4$ AWGRs, as shown in fig. 4; this modular setup is easier to realise. Similarly, by deploying just a single input and output fibre, a label-controlled add/drop node can be constructed with dropand-continue functionality. 


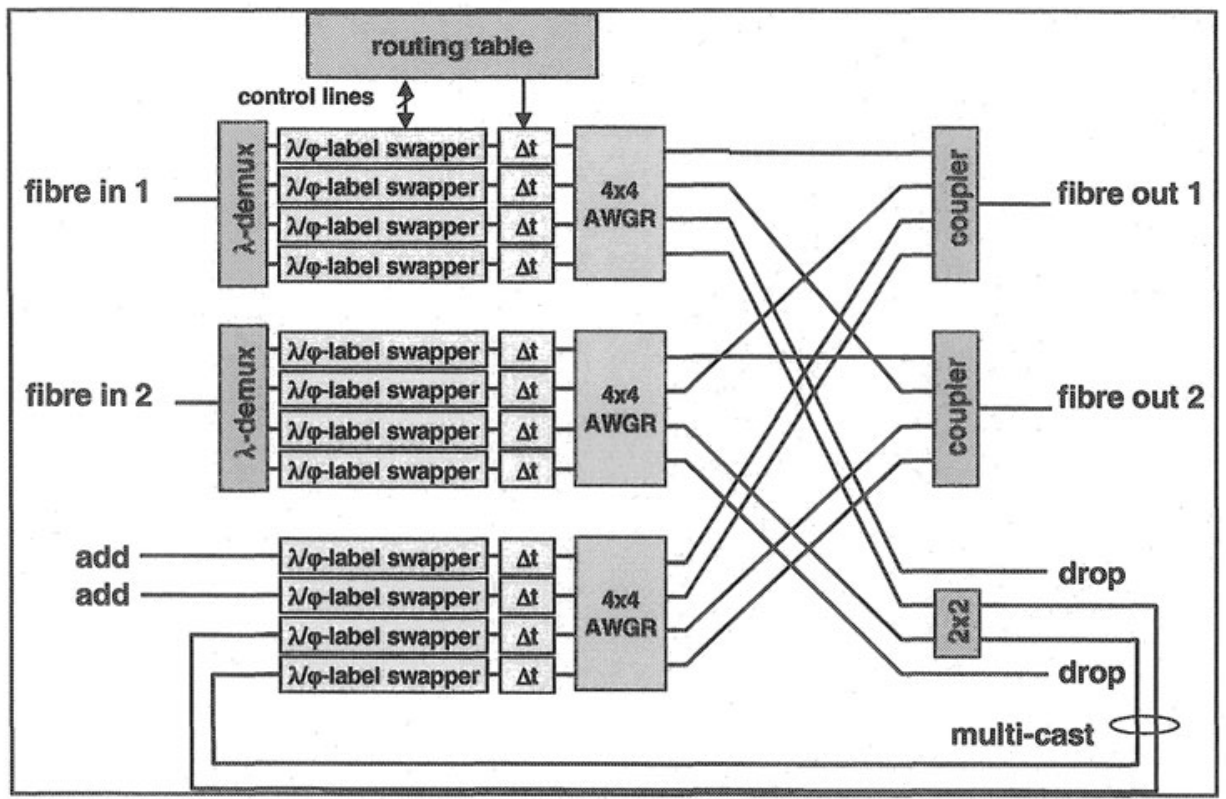

Figure 4. $2 \times 2$ Label-controlled router with $4 \times 4$ AWGRs

\section{TWO-LABEL SWAPPER}

The proposed setup of the wavelength-and-phase information swapper is shown in Fig. 5. A small part of the input signal power is fed to the label processing circuit, which subsequently sets a new wavelength label by means of a tunable laser diode. Via an optical delay line (which compensates for the delay time in the label processor), the payload data is fed into a wavelength converter built with a Mach Zehnder Interferometer (MZI) equipped with Semiconductor Optical Amplifiers (SOAs) in its arms. By means of cross phase modulation in the SOAs induced by the amplitude modulation of the data payload, the data packet is transposed to the new wavelength label. The phase information contained in the DPSK label, however, is not contributing to the cross phase modulation process, and therefore is not transferred. Thus a new DPSK label can be written, by means of the phase modulator integrated together with the MZI wavelength converter in a single InP chip. 


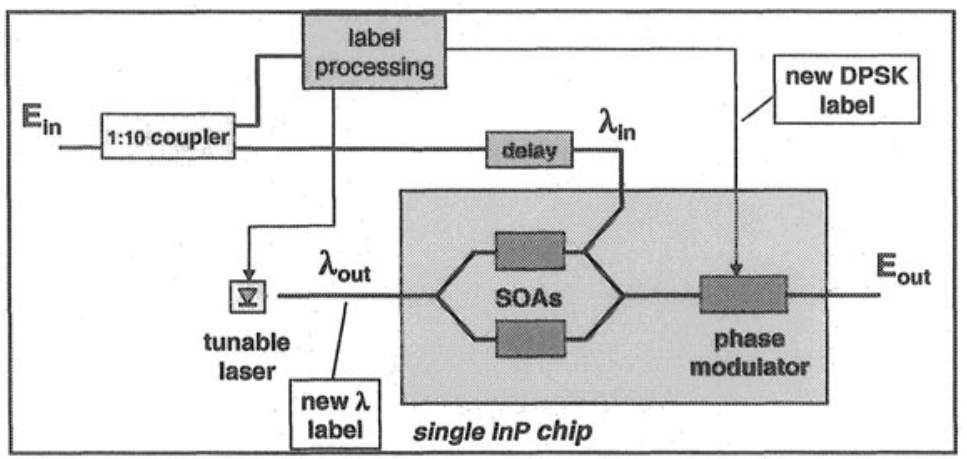

Figure 5. Two-level label swapper

In case of FSK labeling, the FSK label of the input signal is lost in a similar way in thewavelength conversion stage, and a new FSK label can be assigned by modulating the tunable laser's frequency. First simulations using the VPI software package have been done to assess the performance of the label swapper. Due to the interferometric process, careful adjustment of the signal input power and of the injection current of the SOAs is needed for a good wavelength conversion. A wider input power dynamic range requires an input power control stage, e.g. an optical pre-amplifier with automatic gain control.

\section{COMBINED MODUALTION FORMATS}

Two main approaches have been investigated for labeling of optical signals: bit serial labels and out-of-band coding using sub-carrier multiplexed signals. The former adds a fixed bit rate, time multiplexed, label in front of the IP packet payload while the later multiplexes the label information on a RF sub-carrier using the same wavelength of the IP packet payload [2]. In this paper, we present a new approach: orthogonal modulation of the label information with respect to the modulation format of the payload. Two schemes of orthogonal modulation are considered: firstly, ASK modulation for the payload and FSK modulation for the label information. Secondly, DPSK modulation for the label information and ASK modulation for the payload. The former is called the combined ASK/FSK modulation format and later is the combined ASK/DPSK modulation format. These orthogonal modulation schemes are exemplified in Fig.6. 


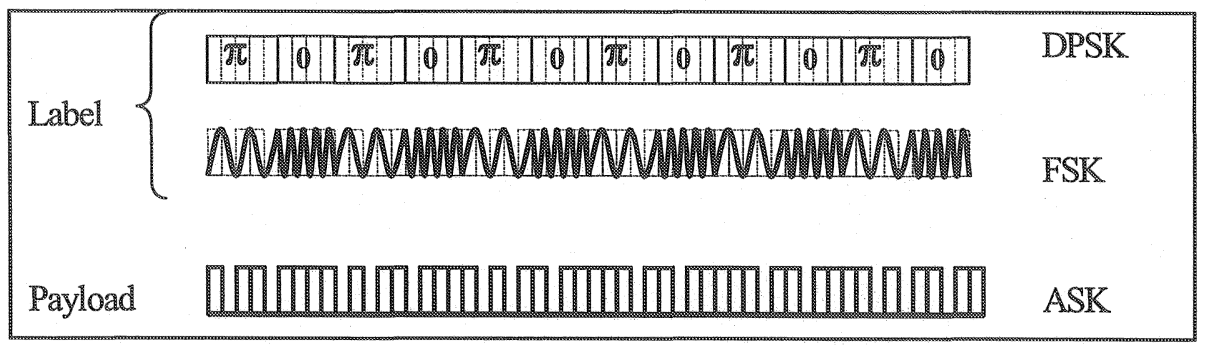

Figure 6. Combined ASK modulation for the payload and orthogonal DPSK or FSK modulation for the label.

\subsection{ASK/FSK Modulation Format}

A block diagram of the ASK/FSK transmitter system is presented in Fig. 7. It is important to note that the value of the modulation depth of the ASK data is crucial for the performance of FSK receiver. The reason being that for any ASK bit-pattern combination there should be enough optical power for the FSK signal to be correctly detected.

Laser light source is directly modulated to obtain FSK modulation. The laser is biased to produce an optical frequency representing a bit ' 0 ' and a small current increment is used to shift the frequency by the deviation frequency $\left(f_{d}\right)$ to represent a bit ' 1 '.

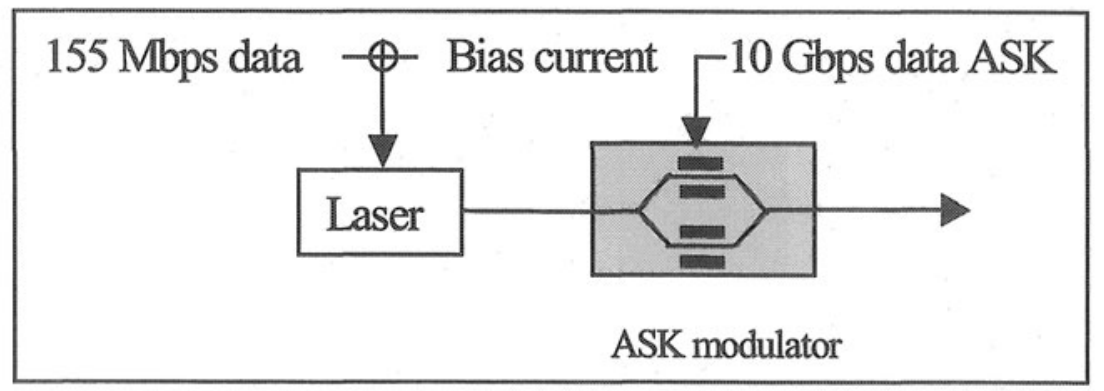

Figure 7. A block diagram of the ASK/FSK transmitter.

Direct detection of the FSK modulation format is performed by conversion of FSK to ASK by filtering one of the spectrum sidebands. A narrow bandpass optical filter is used for this purpose. A block diagram of the FSK receiver is shown in Fig. 8.

We have used VPI software to simulate the performance of combined amplitude and frequency modulation format. The IP payload data rate is 10 Gbits/s while the IP label data rate is $155 \mathrm{Mbits} / \mathrm{s}$. The ASK data is 
generated by using a pseudo random bit sequence (PRBS) with periodic length of $2^{23}-1$. The label information is also generated by using a PRBS with periodic length of $2^{7}-1$.

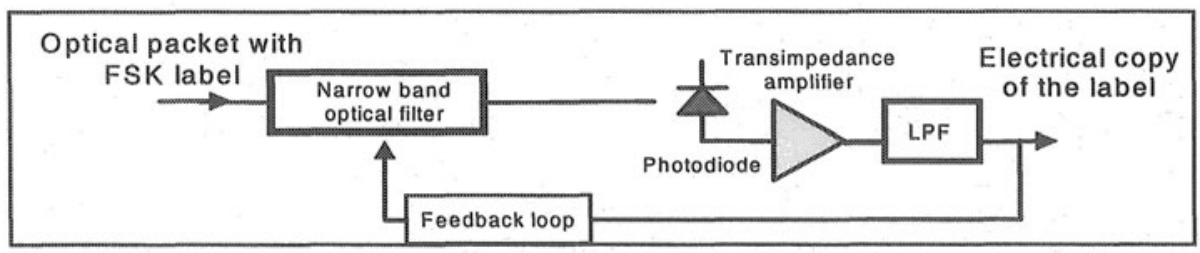

Figure 8. A block diagram of FSK receiver

Fig. 9 and Fig. 10 show the simulation results for the receiver sensitivity as a function of the modulation depth for different values of the laser linewidth and several values of the frequency deviation, respectively. Receiver sensitivity is defined as the average received signal power required to achieve a bit error rate (BER) of $10^{-9}$.

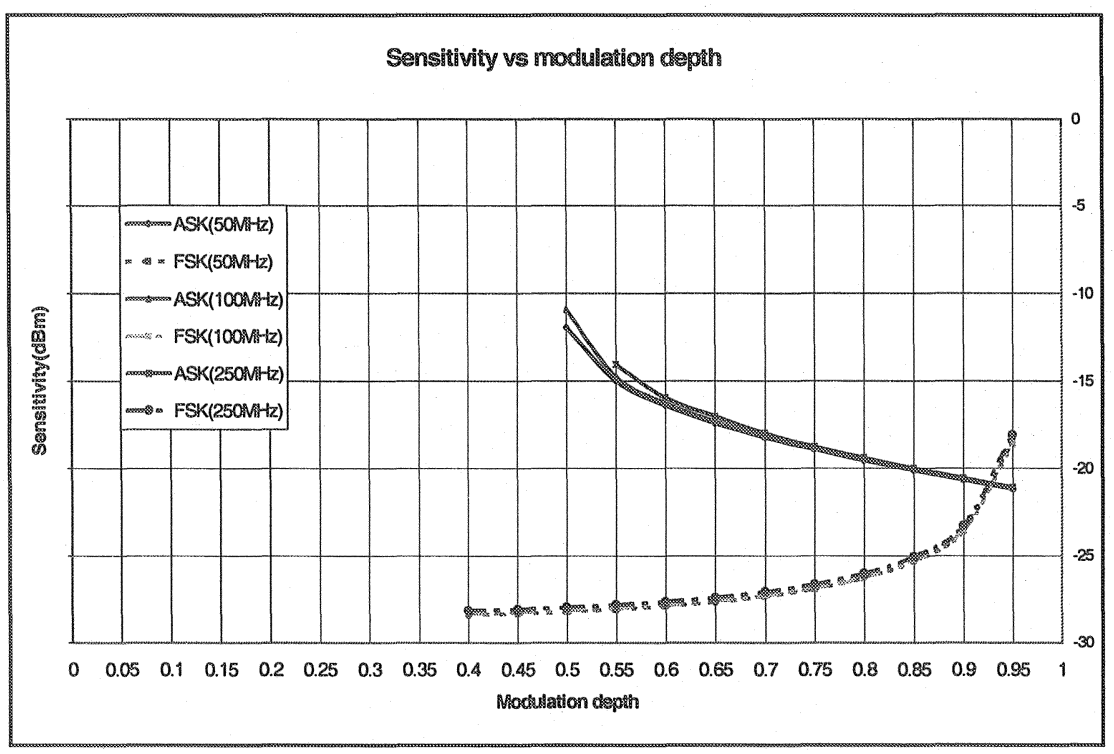

Figure 9. Receiver sensitivity as a function of the ASK modulation depth for different values of the laser line-width and a fixed frequency deviation of $20 \mathrm{GHz}$.

In the simulation, the transmission link is composed of $60 \mathrm{~km}$ of single mode fiber (SMF) and $9.6 \mathrm{~km}$ of dispersion-compensating fiber (DCF). DCF 
is added to compensate for dispersion, and thus to mitigate the effect of amplitude to frequency conversion due to fiber dispersion.

From Fig. 9, it can be seen that the laser line-width does not influence the performance of both ASK and FSK receivers. An optimum operating value of the modulation depth is found around 0.93 for a sensitivity of $-21 \mathrm{dBm}$ for both ASK and FSK receivers.

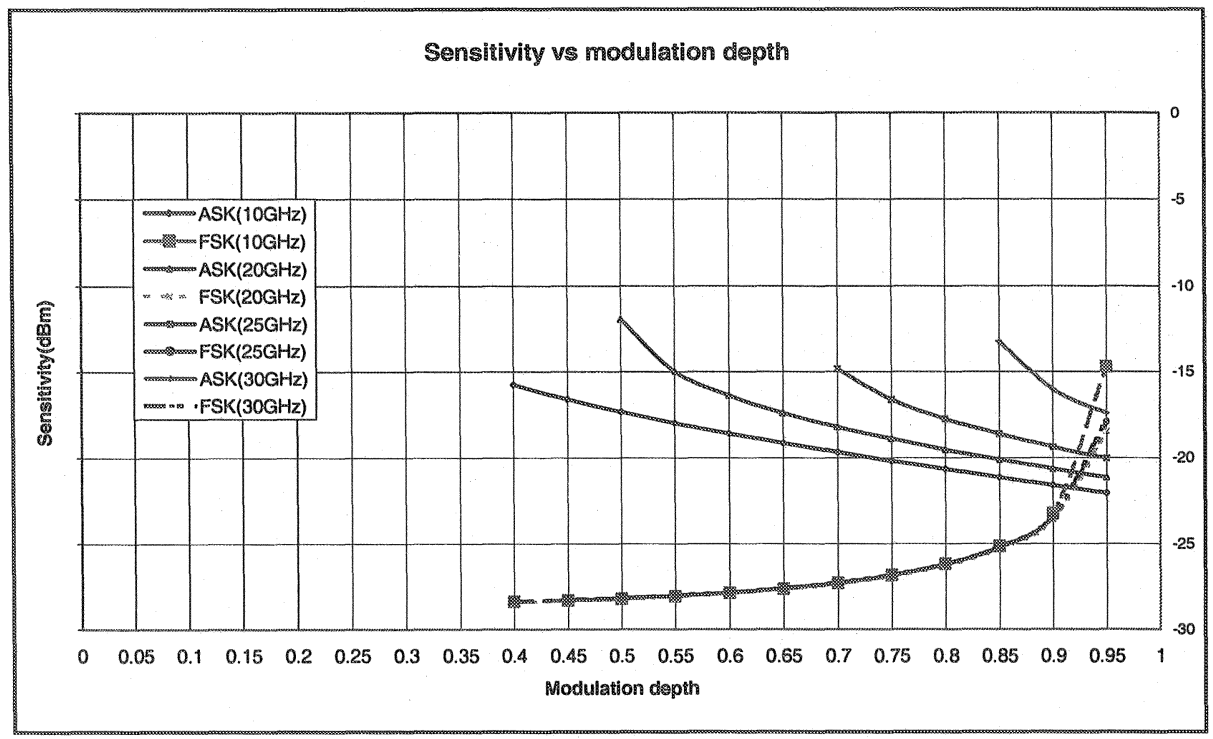

Figure 10. Receiver sensitivity as a function of modulation depth for different values of the frequency deviation and a fixed laser line-width of $50 \mathrm{MHz}$.

We can see from Fig. 10 that for a system with a frequency deviation ranging from $10 \mathrm{GHz}$ to $25 \mathrm{GHz}$, the optimum modulation depth lies between 0.9 and 0.95 . In this case, the receiver sensitivity is between -20 to $-23 \mathrm{dBm}$ for both ASK and FSK receivers. The ASK receiver does not work properly for a frequency deviation of $30 \mathrm{GHz}$ or higher.

The influence of the length of the SMF without DCF on the receiver sensitivity is shown in Fig. 11. The sensitivity of the ASK receiver is largely determined by the fiber dispersion. Due to fiber dispersion, FSK to ASK conversion takes place during propagation, degrading the receiver sensitivity. The performance of the FSK receiver is not influenced by the fiber dispersion. 


\subsection{ASK/DPSK modulation format}

A block diagram of the transmitter for the combined amplitude and differential phase shift keying (DPSK) modulation system is presented in Fig. 12. ASK modulation format is imposed by using an external amplitude modulator. An external phase modulator is used to impose the DPSK modulation format.

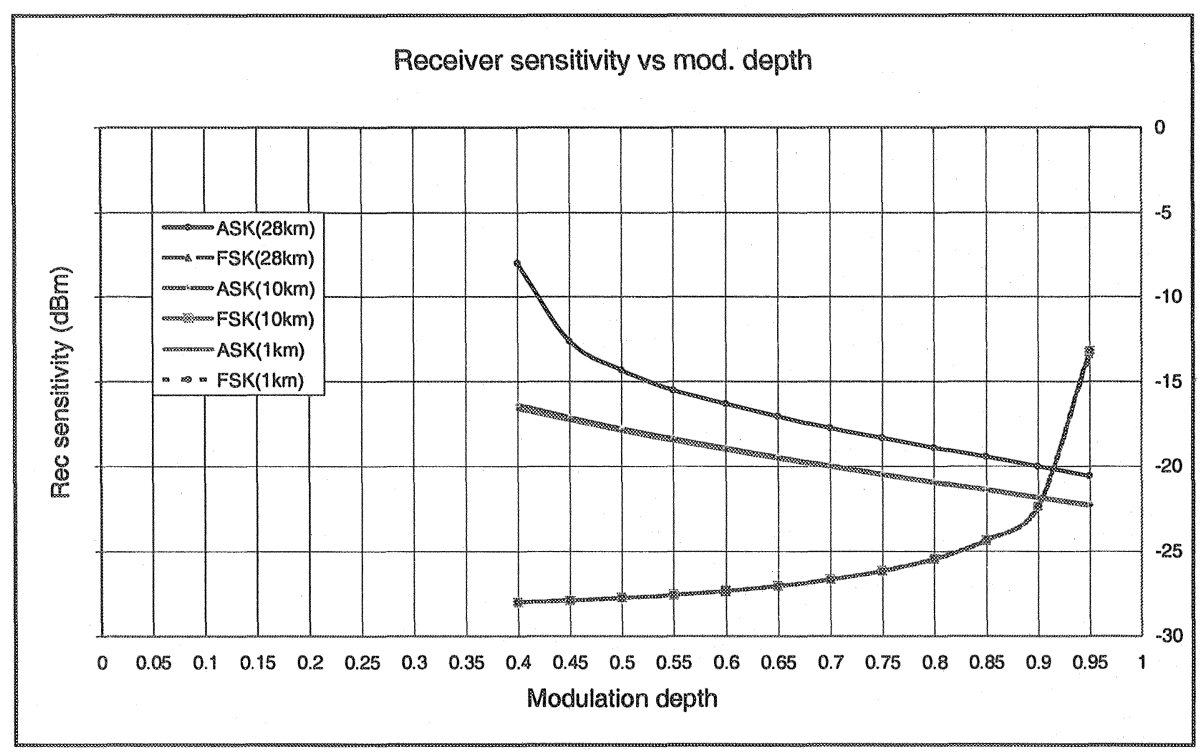

Figure 11. Receiver sensitivity as a function of modulation depth for different SMF lengths at fixed laser line-width and frequency deviation of $25 \mathrm{MHz}$ and $10 \mathrm{GHz}$, respectively.

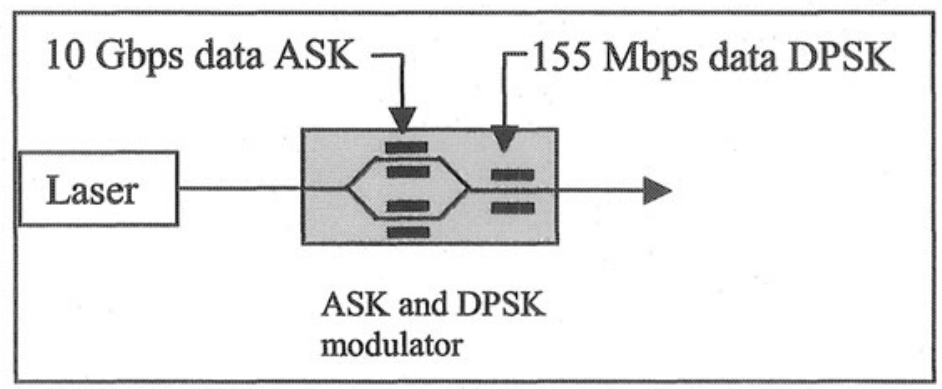

Figure 12. A block diagram of ASK/DPSK transmitter.

It is important to note that the value of the modulation depth of the ASK data is crucial for the performance of the DPSK receiver. The reason being 
that for any ASK bit-pattern combination there should be enough optical power for the DPSK signal to be correctly detected.

We use direct detection for both ASK and DPSK receivers. Figure 13 shows the block diagram for a DPSK receiver with balance photodiodes. In this configuration, the threshold voltage can be set to zero.

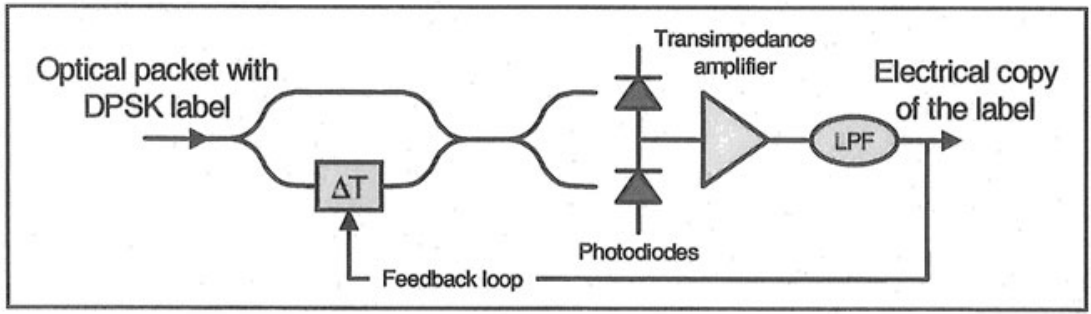

Figure13. The block diagram of DPSK receiver

We have used VPI software for simulation of combined amplitude and differential phase modulation transmission systems. Fig. 14 presents the simulation result for a combined ASK/DPSK transmission system operating at $10 \mathrm{Gbit} / \mathrm{s}$ for the payload and at $155 \mathrm{Mbit} / \mathrm{s}$ for the label information for several values of laser line-width. The data ASK is generated by using a pseudo random bit sequence (PRBS) with periodic length of $2^{23}-1$. The label information is also generated by using a PRBS with periodic length of $2^{7}-1$. Similar to the combined ASK/FSK, the transmission link is composed of 60 $\mathrm{km}$ of single mode fiber (SMF) and $9.6 \mathrm{~km}$ of dispersion-compensating fiber (DCF) in order to compare the performance of both combined ASK/DPSK and ASK/FSK modulation systems.

Fig. 14 shows that the DPSK receiver requires a narrow laser line-width. If the laser line-width is larger than $7 \mathrm{MHz}$, the DPSK receiver does not work properly. However, the combined ASK/DPSK modulation format provides a large range of values for the optimum modulation depth, ranging from 0.6 to 0.96 , that yield the same receiver sensitivity for both ASK and DPSK receivers. If we decrease the modulation depth, starting from 0.96 to 0.6 , then a power penalty of $3.5 \mathrm{~dB}$ is incurred.

The influence of the length of the SMF on the receiver sensitivity is shown in figure 15. We can see that the combined ASK/DPSK modulation format with laser line-width of $2.5 \mathrm{MHz}$ is not influenced largely by the fiber dispersion. 


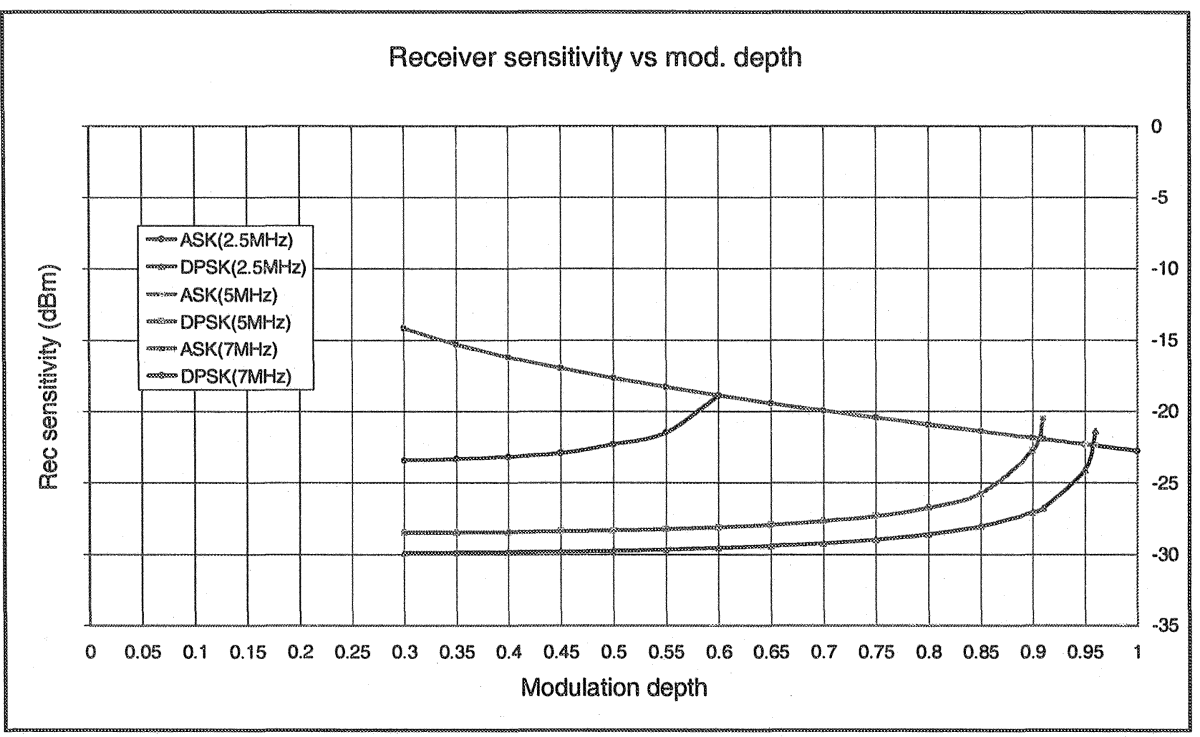

Figure 14. Receiver sensitivity as a function of modulation depth for different values of the laser line-width.

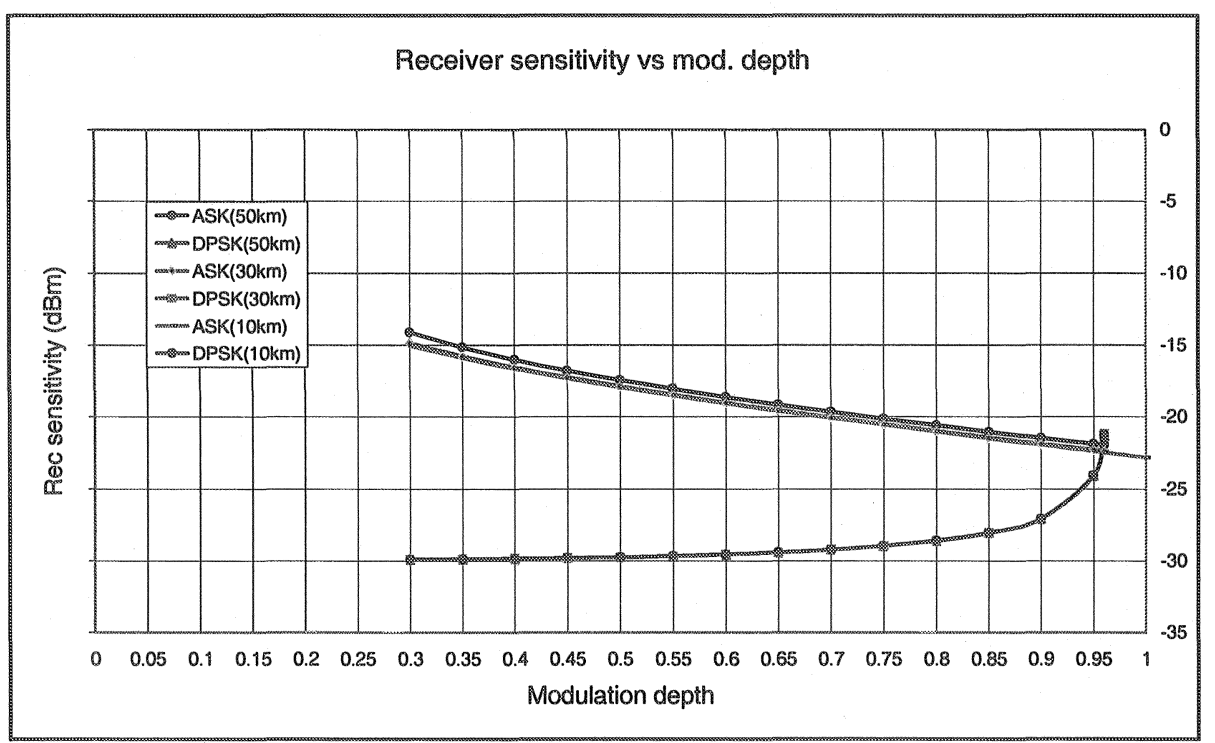

Figure 15. Receiver sensitivity as a function of modulation depth for different SMF lengths and a fixed laser line-width of $2.5 \mathrm{MHz}$. 


\section{DISCUSSIONS}

In this section, we compare the performance of both combined ASK/FSK and ASK/DPSK modulation formats for optically labelled signals. Both the ASK/FSK and ASK/DPSK modulation formats have roughly the same receiver sensitivity, around $-21 \mathrm{dBm}$, for direct detection. However, the combined ASK/FSK modulation format has not a stringent requirement on the value of the laser line-width while the combined ASK/DPSK is very sensitive on that parameter. For example, the ASK/FSK system is able to show a good performance even with a value of $250 \mathrm{MHz}$ for the laser linewidth, while the ASK/DPSK system is only able to operate for values of the laser line-width less than $7 \mathrm{MHz}$. This simulation result indicates that the ASK/FSK system outperforms the ASK/DPSK system with respect to relaxing the requirement on the value of the laser line-width.

ASK/DPSK modulation format provides a large range of operating modulation depth values, starting from 0.6 to 0.96 , while the ASK/FSK system offers a sharp range of values around 0.9 . In practice, a high modulation depth is more difficult to be realized than a relatively low one.

ASK/FSK transmission is much more affected by phase-to-intensity conversion due to dispersion in standard single-mode optical fiber. The simulated ASK/DPSK transmission system can reach a transmission distance of $50 \mathrm{~km}$ (at a laser line-width of $2.5 \mathrm{MHz}$ ) with no performance degradation while ASK/FSK transmission (at a laser line-width of $25 \mathrm{MHz}$ and frequency deviation of $10 \mathrm{GHz}$ ) is limited to $30 \mathrm{~km}$.

Demodulation of FSK encoded data is simpler than demodulation of optical encoded DPSK. FSK can be directly detected by using a bandpass optical filter while DPSK demodulation requires the use of a stable MachZenhder interferometer configuration.

From figure 5, it can be seen that the ASK/FSK modulation format does not require the use of external modulator because direct modulation of a tunable laser can be used to obtain modulation. In an ASK/DPSK system, an external phase modulator is still required. However, FSK modulation format involves residual intensity modulation, which may result in performance degradation of the system.

\section{CONCLUSIONS}

Two-level optical labeling significantly increases the routing and forwarding efficiency of $\mathbb{P}$-over-WDM networks. End-to-end transparent optical paths can be supported by applying both a wavelength label and a label in a modulation format orthogonal to that of the payload. 
Regarding receiver sensitivity and transmission properties, in comparison with the combined ASK/DPSK modulation format, the combined ASK/FSK modulation format is easier to implement and it tolerates a much larger laser line-width, but also requires a more careful fiber dispersion compensation in order to reduce the impact of the phase-to-intensity conversion.

We have presented the performance of both modulation schemes based on the receiver sensitivity and transmission properties. However, for a more comprehensive comparison of both modulation formats for optically labelled signals, other aspects have to be taken into account. For instant, the architecture and the control of the optical label swapper nodes for both modulation formats, scalability with respect to wavelength conversion for both modulation formats, etc. Work is in progress to clarify the issues mentioned above.

\section{ACIKNOWLEDGEMENTS}

This work is done in the IST project STOLAS. The other partners are gratefully acknowledged for their inputs: ADC Altitun, Telenor, COM Institute-TU Denmark, and Univ. College Dublin. The European Commission is acknowledged for accepting the project for partial funding. VPI software was used for the system simulations.

\section{RERERENCES}

[1] N. Ghani, 'Lambda-labeling: A framework for IP-over-WDM using MPLS', Optical Networks Magazine, pp. 45-58, April 2000

[2] D.J. Blumenthal et al., "All-optical label swapping networks and technologies", IEEE J. of Lightwave Technol., Dec. 2000, pp. 2058-2075

[3] Ton Koonen et al., "Optical packet routing in IP-over-WDM networks deploying twolevel optical labeling", Proc. of ECOC'01, Amsterdam, Sep. 30-Oct. 4, 2001, paper Th.L.2.1 